\title{
Large deviations for flows of interacting Brownian motions
}

\author{
A.A.Dorogovtsev, O.V.Ostapenko
}

\begin{abstract}
We establish the large deviation principle (LDP) for stochastic flows of interacting Brownian motions. In particular, we consider smoothly correlated flows, coalescing flows and Brownian motion stopped at a hitting moment.
\end{abstract}

Key words: Large deviations, stochastic flow, Arratia's flow, stochastic differential equation with interaction.

AMS subject classification: Primary 60F10; secondary 37L55.

\section{Introduction}

The article is devoted to the large deviations principle for stochastic flows of Brownian motions on $\mathbb{R}$. We use the following definition.

Definition 1.1. A random field $\{x(u, t) ; u \in \mathbb{R}, t \geq 0\}$ is a stochastic flow of Brownian motions if the following conditions hold:

1 ) for every $u \in \mathbb{R} x(u, \cdot)$ is a Wiener martingale with respect to a common filtration and $x(u, 0)=u$,

2) for every $u_{1} \leq u_{2}$ and $t \geq 0$

$$
x\left(u_{1}, t\right) \leq x\left(u_{2}, t\right) .
$$

Such stochastic flows can be constructed via different ways [1 - 3]. Here we study flows produced by solutions of stochastic differential equations, and their limits. Let $W$ be a Wiener sheet on $\mathbb{R} \times[0 ;+\infty)$ (i.e. $W$ is the Gaussian random measure with independent values on disjoint sets and the Lebesgue measure as a control measure). Consider the equation

$$
\left\{\begin{array}{l}
d x(u, t)=\int_{\mathbb{R}} \varphi(x(u, t)-p) W(d p, d t), \\
x(u, 0)=u, u \in \mathbb{R}
\end{array}\right.
$$


with a smooth function $\varphi \in S$ (S denotes the Schwartz space). Suppose, that

$$
\int_{\mathbb{R}} \varphi^{2}(p) d p=1 .
$$

Using an orthonormal basis $\left\{e_{n} ; n \geq 1\right\}$ in $L_{2}(\mathbb{R}),(1.1)$ can be rewritten as a $\mathrm{SDE}$ with respect to the countable family of independent Wiener processes

$$
\beta_{n}(t)=\int_{0}^{t} \int_{\mathbb{R}} e_{n}(p) W(d p, d s), n \geq 1 .
$$

In such terms (1.1) takes the form

$$
\left\{\begin{array}{l}
d x(u, t)=\sum_{n=1}^{\infty} a_{n}(x(u, t)) d \beta_{n}(t), \\
x(u, 0)=u, u \in \mathbb{R} .
\end{array}\right.
$$

Under our conditions on $\varphi$ Equation (1.1) has a unique solution, which is a flow of diffeomorphisms [3,5]. Consider the properties of $x$. Note that for arbitrary $u_{1} \leq u_{2}$ and $t \geq 0$

$$
x\left(u_{1}, t\right) \leq x\left(u_{2}, t\right)
$$

(diffeomorphic property). For every $u x(u, \cdot)$ is a continuous martingale with the characteristics

$$
\langle x(u, \cdot)\rangle_{t}=\int_{0}^{t} \int_{\mathbb{R}} \varphi^{2}(x(u, s)-p) d p d s=t, t \geq 0 .
$$

Consequently [4], $x(u, \cdot)$ is a Brownian motion, starting from $u$. Note that for different points $u_{1}, u_{2}$ the processes $x\left(u_{1}, \cdot\right)$ and $x\left(u_{2}, \cdot\right)$ are correlated:

$$
\begin{gathered}
\left\langle x\left(u_{1}, \cdot\right), x\left(u_{2}, \cdot\right)\right\rangle_{t}= \\
=\int_{0}^{t} \int_{\mathbb{R}} \varphi\left(x\left(u_{1}, s\right)-p\right) \varphi\left(x\left(u_{2}, s\right)-p\right) d p d s= \\
=\int_{0}^{t} \Phi\left(x\left(u_{1}, s\right)-x\left(u_{2}, s\right)\right) d s,
\end{gathered}
$$

where

$$
\Phi(r)=\int_{\mathbb{R}} \varphi(r-p) \varphi(p) d p .
$$

The function $\Phi$ can be treated as a momental correlation between different one-point motions in the flow (1.1) [3]. The singular case can be obtained from (1.1) when $\varphi^{2}$ tends to $\delta_{0}$. More precisely, the following result was proved in [5]. 
Theorem 1.1. Let $\varphi_{\varepsilon} \in S, \operatorname{supp} \varphi_{\varepsilon} \subset[-\varepsilon ; \varepsilon]$,

$$
\int_{\mathbb{R}} \varphi_{\varepsilon}^{2}(p) d p=1
$$

$\varphi_{\varepsilon}^{2} \rightarrow \delta_{0}, \varepsilon \rightarrow 0+$. Then the n-pont motions of the flow (1.1) with $\varphi_{\varepsilon}$ instead of $\varphi$ converge in distribution to the n-point motions of Arratia's flow.

Recall, that Arratia's flow consists of Brownian motions, which are independent up to their meeting and move together after that [2]. This flow can be treated as a Brownian flow with $\delta_{0}$-correlation.

A recent result concerning the LDP for stochastic flows of diffeomorphisms is due to A. Budhiraja, D. Dupuis and V. Maroulas [6]. These authors considered stochastic dynamical systems driven by an infinite-dimensional Brownian motion

$$
\begin{aligned}
& d \phi_{s, t}^{\varepsilon}(x)=F^{\varepsilon}\left(\phi_{s, t}^{\varepsilon}(x), d t\right), \\
& \phi_{s, s}^{\varepsilon}(x)=x, 0 \leq s \leq t \leq T, \quad x \in \mathbb{R}^{d},
\end{aligned}
$$

where $F^{\varepsilon}(x, t)$ is a $C^{k+1}$-Brownian motion in the sence of Kunita [3]. They got the LDP for $\left(\phi^{\varepsilon}, F^{\varepsilon}\right)_{\varepsilon>0}$ in the space $C\left([0, T] ; C^{m}\left(\mathbb{R}^{d}\right)\right)$ and $C\left([0, T] ; G^{m}\right)$ $\left(G^{m}\right.$ is the group of $C^{m}$-diffeomorphisms on $\left.\mathbb{R}^{d}\right)$. The proof of the LDP is based on variational representations for functionals of infinite-dimensional Brownian motion [8].

Note that the family $\left\{x^{\varepsilon}\right\}_{\varepsilon>0}$ in (1.1) can be represented as the flow described in [6]. Indeed, we can write

$$
\begin{gathered}
d x^{\varepsilon}(u, t)=\sqrt{\varepsilon} \sum_{k=1}^{\infty} a_{k}\left(x^{\varepsilon}(u, t)\right) d \beta_{k}(t), \\
x^{\varepsilon}(u, 0)=u, t \in[0 ; 1], u \in \mathbb{R},
\end{gathered}
$$

where

$$
\begin{gathered}
a_{k}(v)=\int_{\mathbb{R}} \varphi(v-p) e_{k}(p) d p, \\
\beta_{k}(t)=\int_{0}^{t} \int_{\mathbb{R}} e_{k}(p) W(d p, d s),
\end{gathered}
$$

$\left\{e_{k}, k \geq 1\right\}$ is an orthonormal basis in $L_{2}(\mathbb{R})$. However, in our article we present the LDP not only for smoothly correlated, but also for coalescing 
flows. In particular, we prove the LDP for Arratia's flow. The main difference between smooth and singular correlation is the presence of $W$ in (1.1). Really, traditionally one can get the LDP for (1.1) from $W$ (roughly speaking). But Arratia's flow does not contain a white noise. Hence we will need an additional construction. The article is organized as follows. In the second part we prove the LDP for smooth case (1.1) using a method different from that of [6]. The third part is devoted to the structure of Arratia's flow, which is a limit case of (1.1). Here we present some facts about the total time of free motion for particles in this flow. The fourth part is devoted to the LDP for Brownian motion stopped at a hitting moment. Here the time scaling is used instead of the space scaling. The fifth part contains the LDP for the $n$-point motions of Arratia's flow. Finally the last part of the article deals with the LDP for Arratia's flow in the Lévy-Prokhorov distance.

\section{LDP for smoothly correlated flows}

For $\varepsilon>0$ let $x^{\varepsilon}$ be the stochastic flow described by the differential equation

$$
\begin{aligned}
& d x^{\varepsilon}(u, t)=\sqrt{\varepsilon} \int_{\mathbb{R}} \varphi\left(x^{\varepsilon}(u, t)-p\right) W(d p, d t), \\
& x^{\varepsilon}(u, 0)=u, u \in \mathbb{R}, t \in[0,1] .
\end{aligned}
$$

For every $\varepsilon>0 \quad x^{\varepsilon}$ is a flow of homeomorphisms in $\mathbb{R}[3]$. Moreover, one can get the following relations describing the growth of $x^{\varepsilon}$ with respect to spatial variable

$$
\forall \delta>0: \quad \lim _{|u| \rightarrow+\infty} \frac{\left|x^{\varepsilon}(u, t)\right|}{1+|u|^{\delta+1}}=\lim _{|u| \rightarrow+\infty} \frac{|u|}{1+\left|x^{\varepsilon}(u, t)\right|^{\delta+1}}=0 \text { a.s. }
$$

It follows from this property, that $x^{\varepsilon}$ can be considered as a random element in the space $\mathfrak{X}=C\left([0 ; 1] ; L_{2}(\mathbb{R}, \mu)\right)$. Here $\mu$ is the standard normal distribution on $\mathbb{R}$. We suppose, that $\mathfrak{X}$ is equipped with the norm

$$
\mathfrak{X} \ni x \mapsto\|x\|=\sup _{t \in[0 ; 1]}\left(\int_{\mathbb{R}} x(u, t)^{2} \mu(d u)\right)^{\frac{1}{2}} .
$$

We are going to establish the LDP for $x^{\varepsilon}$ in the space $\mathfrak{X}$. The main result is based on an extension of the contraction principle to maps that are not continuous, but can be approximated well by continuous maps [9]. Define 
an approximation for $x^{\varepsilon}$ as follows. Let $x_{m}^{\varepsilon}, m \geq 1$ be the stochastic flow described by the equation

$$
\left\{\begin{array}{l}
d x_{m}^{\varepsilon}(u, t)=\sqrt{\varepsilon} \int_{\mathbb{R}} \varphi\left(x_{m}^{\varepsilon}\left(u, \frac{[t m]}{m}\right)-p\right) W(d p, d t) \\
x_{m}^{\varepsilon}(u, 0)=u, u \in \mathbb{R}, t \in[0 ; 1]
\end{array}\right.
$$

in which the coefficients of (2.1) are frozen over the time intervals $\left[\frac{k}{m}, \frac{k+1}{m}\right), k=$ $0, \ldots, m-1$. Consider first the case $m=1$. In this case (2.4) has the form

$$
\left\{\begin{array}{l}
d y^{\varepsilon}(u, t)=\sqrt{\varepsilon} \int_{\mathbb{R}} \varphi(u-p) W(d p, d t), \\
y^{\varepsilon}(u, 0)=u, u \in \mathbb{R}, t \in[0 ; 1] .
\end{array}\right.
$$

Note that $y^{\varepsilon}$ is a Gaussian random element in $\mathfrak{X}$ obtained from $W$. So the LDP for the family $y^{\varepsilon}$ has a known form $[10,11]$. Define $H$ as the set of functions of the type

$$
\begin{gathered}
h(u, t)=u+\int_{0}^{t} \int_{\mathbb{R}} \varphi(u-p) a(p, s) d p d s, \\
a \in L_{2}(\mathbb{R} \times[0 ; 1]), u \in \mathbb{R}, t \in[0 ; 1] .
\end{gathered}
$$

Also, let $\mathcal{F}$ be the Fourier transform on $\mathbb{R}$.

Theorem 2.1. Let $\varphi \in S$ be such, that $\mathcal{F}(\varphi) \neq 0$ a.s. Then the family $\left\{y^{\varepsilon}\right\}$ satisfies the LDP in $\mathfrak{X}$ with rate function

$$
I_{y}(h)=\left\{\begin{array}{l}
\frac{1}{4 \pi} \int_{0}^{1} \int_{\mathbb{R}}\left(\frac{\mathcal{F}(\dot{h}(\cdot, t))(\lambda)}{\mathcal{F}(\varphi)(\lambda)}\right)^{2} d \lambda d t, h \in H, \\
\infty, h \notin H
\end{array}\right.
$$

i.e.:

1) for each closed set $F \subset \mathfrak{X}$

$$
\varlimsup_{\varepsilon \rightarrow 0} \varepsilon \log P\left\{y^{\varepsilon} \in F\right\} \leq-\inf _{f \in F} I(f) ;
$$

2) for each open set $G \subset \mathfrak{X}$

$$
\varliminf_{\varepsilon \rightarrow 0} \varepsilon \log P\left\{y^{\varepsilon} \in G\right\} \geq-\inf _{f \in G} I(f) .
$$


Proof. Note that

$$
y(u, t)=u+\int_{0}^{t} \int_{\mathbb{R}} \varphi(u-p) W(d p, d s), t \in[0,1], u \in \mathbb{R},
$$

is a Gaussian random element in $\mathfrak{X}$. Define the map $i: L_{2}(\mathbb{R} \times[0 ; 1]) \rightarrow \mathfrak{X}$ as

$$
i(a)(u, t)=u+\int_{0}^{t} \int_{\mathbb{R}} \varphi(u-p) a(p, s) d p d s, a \in L_{2}(\mathbb{R} \times[0 ; 1]) .
$$

$i$ is a linear continuous operator. Note that due to the condition on the Fourier transform of $\varphi$ the operator $i$ is injection.

Since $W$ can be considered as a generalized Gaussian random element in $L_{2}(\mathbb{R} \times[0 ; 1])$ with zero mean and identity covariation, then by the standard arguments one can verify, that covariation of $y$ is $i i^{*}$. Therefore $[10,11],\left\{y^{\varepsilon}\right\}$ satisfies the LDP in $\mathfrak{X}$ with rate function

$$
I_{y}(h)=\left\{\begin{array}{l}
\frac{1}{2}\left\|i^{-1}(h)\right\|_{L_{2}(\mathbb{R} \times[0 ; 1])}^{2}, h \in H, \\
\infty, h \notin H .
\end{array}\right.
$$

Rewrite the rate function in terms of the Fourier transform. Let $h=i(a)$, then

$$
\dot{h}(u, t)=\int_{\mathbb{R}} \varphi(u-p) a(p, t) d p .
$$

For fixed $t \in[0,1]$ apply the Fourier transform:

$$
\mathcal{F}(\dot{h}(\cdot, t))=\mathcal{F}(\varphi) \mathcal{F}(a(\cdot, t)) .
$$

Hence,

$$
\left\|i^{-1}(h)\right\|_{L_{2}(\mathbb{R} \times[0 ; 1])}^{2}=\frac{1}{2 \pi} \int_{0}^{1} \int_{\mathbb{R}}\left(\frac{\mathcal{F}(\dot{h}(\cdot, t))(\lambda)}{\mathcal{F}(\varphi)(\lambda)}\right)^{2} d \lambda d t .
$$

The theorem is proved.

The contraction principle [9] yields the LDP for $\left\{y^{\varepsilon}(\cdot, t)\right\}$ at a fixed time $t \in[0,1]$.

Corollary 2.1. For any fixed $t \in[0,1]$ the family $\left\{y^{\varepsilon}(\cdot, t)\right\}$ satisfies the LDP in $L_{2}(\mathbb{R}, \mu)$ with rate function

$$
I_{t}(h)=\left\{\begin{array}{l}
\frac{1}{4 \pi t} \int_{\mathbb{R}}\left(\frac{\mathcal{F}(h)(\lambda)}{\mathcal{F}(\varphi)(\lambda)}\right)^{2} d \lambda, h \in H_{t} \\
\infty, h \notin H_{t}
\end{array}\right.
$$


where $H_{t}$ is the set of functions from $L_{2}(\mathbb{R}, \mu)$ having square-integrable derivative.

Remark. Observe that $y^{\varepsilon}$ is a random element in $\mathcal{Y}=C\left([0,1] ; L_{2}(\mathbb{R}, \nu)\right)$, where $\nu$ is the measure on $\mathbb{R}$ with the density $\frac{1}{1+|u|}, u \in \mathbb{R}, l>3$. And for any $p \geq 2$ and fixed time $t, y^{\varepsilon}(\cdot, t)$ is a random element in $L_{p}(\mathbb{R}, \mu)$. Using the same arguments as in Theorem 2.1 one can show, that $\left\{y^{\varepsilon}\right\}$ satisfies the LDP in $\mathcal{Y}$ and $\left\{y^{\varepsilon}(\cdot, t)\right\}$ satisfies the LDP in $L_{p}(\mathbb{R}, \mu)$ with the same rate functions (2.6) and (2.7) correspondingly.

To prove the LDP for $x_{m}^{\varepsilon}$ and $x^{\varepsilon}$ we need an additional assumption on the function $\varphi$. From now on we will suppose, that the following relation holds:

H1. $\varphi=\psi_{1} * \psi_{2}, \psi_{1}, \psi_{2} \in S$.

In the case $m>1, x_{m}^{\varepsilon}$ can be represented as

$$
\begin{aligned}
& x_{m}^{\varepsilon}(u, t)=x_{m}^{\varepsilon}\left(u, \frac{k}{m}\right)+ \\
& +\sqrt{\varepsilon} \int_{\mathbb{R}} \psi_{1}\left(x_{m}^{\varepsilon}\left(u, \frac{k}{m}\right)-q\right) \int_{\frac{k}{m}}^{t} \int_{\mathbb{R}} \psi_{2}(q-p) W(d p, d s) d q, \\
& t \in\left[\frac{k}{m}, \frac{k+1}{m}\right], k=0, \ldots, m-1 .
\end{aligned}
$$

By the remark $\left\{\sqrt{\varepsilon} \int_{\frac{k}{m}}^{\cdot} \int_{\mathbb{R}} \psi_{2}(\cdot-p) W(d p, d s)\right\}$ satisfies the LDP in $\mathcal{Y}$ and $\left\{\sqrt{\varepsilon} \int_{\frac{k}{m}}^{\frac{k+1}{m}} \int_{\mathbb{R}} \psi_{2}(\cdot-p) W(d p, d s)\right\}$ satisfies the LDP in $L_{p}(\mathbb{R}, \mu)$, for any $p \geq 2$.

Before formulating the main result in this section we will prove some lemmas establishing the continuity property of some maps.

Lemma 2.1. For $f \in L_{p l}(\mathbb{R}, \mu)$ and $g \in L_{2}(\mathbb{R}, \nu), p \geq 2$, define

$$
\mathcal{F}^{p}(f, g)(u)=\int_{\mathbb{R}} \psi_{2}(f(u)-q) g(q) d q .
$$

Then $\mathcal{F}^{p}$ is continuous map from $L_{p l}(\mathbb{R}, \mu) \times L_{2}(\mathbb{R}, \nu)$ to $L_{p}(\mathbb{R}, \mu)$.

Proof. Let $f_{n} \rightarrow f, n \rightarrow \infty$ in $L_{p l}(\mathbb{R}, \mu)$ and $g_{n} \rightarrow g, n \rightarrow \infty$ in $L_{2}(\mathbb{R}, \nu)$. Then

$$
\begin{gathered}
\left\|\mathcal{F}^{p}\left(f_{n}, g_{n}\right)-\mathcal{F}^{p}(f, g)\right\|_{L_{p}(\mu)} \leq \\
\leq\left\|\mathcal{F}^{p}\left(f_{n}, g_{n}\right)-\mathcal{F}^{p}\left(f_{n}, g\right)\right\|_{L_{p}(\mu)}+\left\|\mathcal{F}^{p}\left(f_{n}, g\right)-\mathcal{F}^{p}(f, g)\right\|_{L_{p}(\mu)} \leq
\end{gathered}
$$




$$
\begin{gathered}
\leq\left\|g_{n}-g\right\|_{L_{2}(\nu)}\left[\int_{\mathbb{R}}\left(\int_{\mathbb{R}} \psi_{2}^{2}\left(f_{n}(u)-q\right)\left(1+|q|^{l}\right) d q\right)^{p / 2} \mu(d u)\right]^{1 / p}+ \\
+\|g\|_{L_{2}(\nu)}\left[\int_{\mathbb{R}}\left(\int_{\mathbb{R}}\left(\psi_{2}\left(f_{n}(u)-q\right)-\psi_{2}(f(u)-q)\right)^{2}\left(1+|q|^{l}\right) d q\right)^{p / 2} \mu(d u)\right]^{1 / p} .
\end{gathered}
$$

Observe that for some $C>0$

$$
\int_{\mathbb{R}} \psi_{2}^{2}(f(u)-q)\left(1+|q|^{l}\right) d q \leq C\left(|f(u)|^{l}+1\right) .
$$

Since $f_{n} \rightarrow f, n \rightarrow \infty$ in $L_{p l}(\mathbb{R}, \mu)$, there exists a constant $C_{1}>0$ such that

$$
\left\|f_{n}\right\|_{L_{p l}(\mu)} \leq C_{1}, n \geq 1
$$

So, for some constant $C_{2}>0$

$$
\begin{gathered}
\int_{\mathbb{R}}\left(\int_{\mathbb{R}} \psi_{2}^{2}\left(f_{n}(u)-q\right)\left(1+|q|^{l}\right) d q\right)^{p / 2} \mu(d u) \leq \\
\leq \int_{\mathbb{R}} C^{p / 2}\left(\left|f_{n}(u)\right|^{l}+1\right)^{p / 2} \mu(d u) \leq \\
\leq C^{p / 2}\left(\left\|f_{n}\right\|_{L_{p l}(\mu)}^{l}+1\right)^{p} \leq C_{2}, n \geq 1 .
\end{gathered}
$$

Without loss of generality, we assume that $\left\{f_{n}\right\}$ converges to $f$ almost everywhere. Denote

$$
b_{n}(u, q)=\left(\psi_{2}\left(f_{n}(u)-q\right)-\psi_{2}(f(u)-q)\right)^{p}\left(1+|q|^{l}\right)^{p / 2} .
$$

Let us check, that

$$
\sup _{n \geq 1} \int_{\mathbb{R}} \int_{\mathbb{R}} b_{n}^{2}(u, q) d q \mu(d u)<\infty .
$$

Indeed, for some $C_{3}>0$

$$
\begin{gathered}
\int_{\mathbb{R}} \int_{\mathbb{R}} b_{n}^{2}(u, q) d q \mu(d u) \leq \\
\leq 2^{2 p-1}\left(\int_{\mathbb{R}} \int_{\mathbb{R}} \psi_{2}^{2 p}\left(f_{n}(u)-q\right)\left(1+|q|^{l}\right)^{p} d q \mu(d u)+\right. \\
\left.+\int_{\mathbb{R}} \int_{\mathbb{R}} \psi_{2}^{2 p}(f(u)-q)\left(1+|q|^{l}\right)^{p} d q \mu(d u)\right) \leq
\end{gathered}
$$




$$
\leq 2^{2 p-1} C^{p}\left(\left(\left\|f_{n}\right\|_{L_{p l}(\mu)}^{l}+1\right)^{p}+\left(\|f\|_{L_{p l}(\mu)}^{l}+1\right)^{p}\right) \leq C_{3}, n \geq 1 .
$$

This estimation establishes uniform integrability of $\left\{b_{n}\right\}$ and proves the lemma.

Corollary 2.2. For $f \in L_{2 l}(\mathbb{R}, \mu)$ and $g \in \mathcal{Y}$ define

$$
\mathcal{F}(f, g)(t, u)=\int_{\mathbb{R}} \psi_{2}(f(u)-q) g(t, q) d q, t \in[0 ; 1], u \in \mathbb{R} .
$$

Then $\mathcal{F}$ is continuous map from $L_{2 l}(\mathbb{R}, \mu) \times \mathcal{Y}$ to $\mathfrak{X}$.

The following representation holds for every $k=1, \ldots, m$

$$
\begin{aligned}
& x_{m}^{\varepsilon}\left(u, \frac{k}{m}\right)= \mathcal{F}^{2 l}\left(x_{m}^{\varepsilon}\left(\cdot, \frac{k-1}{m}\right), y^{\varepsilon}\left(\cdot, \frac{k}{m}\right)-y^{\varepsilon}\left(\cdot, \frac{k-1}{m}\right)\right)(u), \\
& x_{m}^{\varepsilon}\left(u, \frac{k-1}{m}\right)=\mathcal{F}^{2 l^{2}}\left(x_{m}^{\varepsilon}\left(\cdot, \frac{k-2}{m}\right), y^{\varepsilon}\left(\cdot, \frac{k-1}{m}\right)-y^{\varepsilon}\left(\cdot, \frac{k-2}{m}\right)\right)(u), \ldots \\
& x_{m}^{\varepsilon}\left(u, \frac{1}{m}\right)=\mathcal{F}^{2 l^{k}}\left(e, y^{\varepsilon}\left(\cdot, \frac{1}{m}\right)\right)(u),
\end{aligned}
$$

where

$$
\begin{gathered}
y^{\varepsilon}(u, t)=\sqrt{\varepsilon} \int_{0}^{t} \int_{\mathbb{R}} \psi_{2}(u-p) W(d p, d s), t \in[0 ; 1], u \in \mathbb{R}, \\
e(u)=u, u \in \mathbb{R} .
\end{gathered}
$$

Consequently, $x_{m}^{\varepsilon}\left(\cdot, \frac{k}{m}\right)$ is the image of $y^{\varepsilon}$ under some continuous map from $\mathcal{Y}$ to $L_{2 l}(\mathbb{R}, \mu)$. By induction one can prove that $x_{m}^{\varepsilon}$ can be represented as the image of $y^{\varepsilon}$ under a continuous map from $\mathcal{Y}$ to $\mathfrak{X}$ and, consequently, satisfy the LDP. We will show that $\left\{x_{m}^{\varepsilon}\right\}$ are exponentially good approximations of $\left\{x^{\varepsilon}\right\}$, i.e. for every $\delta>0$

$$
\lim _{m \rightarrow \infty} \varlimsup_{\varepsilon \rightarrow 0} \varepsilon \log P\left\{\left\|x_{m}^{\varepsilon}-x^{\varepsilon}\right\|>\delta\right\}=-\infty,
$$

where the norm $\|\cdot\|$ is taken in the space $\mathfrak{X}$. Following [9], let us prove some auxiliary results.

Consider the stochastic flow described by the equation

$$
\begin{aligned}
& d z^{\varepsilon}(u, t)=\sqrt{\varepsilon} \int_{\mathbb{R}} \psi(t, u, p) W(d p, d t), \\
& z^{\varepsilon}(u, 0)=\alpha(u), u \in \mathbb{R}, t \in[0 ; 1],
\end{aligned}
$$


where $\alpha \in L_{2}(\mathbb{R}, \mu), \psi \in L_{2}([0,1] \times \mathbb{R} \times \mathbb{R} \times \Omega)$, for every $u \in \mathbb{R} \quad \psi(\cdot, u, \cdot)$ is progressively measurable with respect to the filtration $\mathcal{F}_{t}=\sigma\{w(\Delta), \Delta \subset$ $\mathbb{R} \times[0, t]\}$. For every $t \in[0 ; 1], z^{\varepsilon}(\cdot, t) \in L_{2}(\mathbb{R}, \mu)$ and $\left\|z^{\varepsilon}(\cdot, t)\right\|_{L_{2}(\mu)}^{2}$ has the stochastic differential [3]

$$
\begin{aligned}
d\left\|z^{\varepsilon}(\cdot, t)\right\|_{L_{2}(\mu)}^{2} & =2 \sqrt{\varepsilon} \int_{\mathbb{R}} \int_{\mathbb{R}} z^{\varepsilon}(u, t) \psi(t, u, p) \mu(d u) W(d p, d t)+ \\
& +\varepsilon \int_{\mathbb{R}} \int_{\mathbb{R}} \psi^{2}(t, u, p) \mu(d u) d p d t .
\end{aligned}
$$

Let $\tau \in[0 ; 1]$ be a stopping time with respect to the filtration $\mathcal{F}_{t}$. Suppose, that for some constants $L, \rho$ and any $t \in[0, \tau]$ :

$$
\int_{\mathbb{R}} \int_{\mathbb{R}} \psi^{2}(t, u, p) \mu(d u) d p \leq L\left(\left\|z^{\varepsilon}(\cdot, t)\right\|_{L_{2}(\mu)}^{2}+\rho^{2}\right) .
$$

Lemma 2.2. For any $\delta>0, \varepsilon \leq 1$

$$
\varepsilon \log P\left\{\sup _{t \in[0, \tau]}\left\|z^{\varepsilon}(\cdot, t)\right\|_{L_{2}(\mu)} \geq \delta\right\} \leq L+\log \frac{\rho^{2}+\|\alpha\|_{L_{2}(\mu)}^{2}}{\rho^{2}+\delta^{2}} .
$$

Proof. Let $\Phi(t)=f\left(z^{\varepsilon}(\cdot, t)\right)$, where

$$
f(z)=\left(\rho^{2}+\|z\|_{L_{2}(\mu)}^{2}\right)^{1 / \varepsilon} .
$$

Using the Itô formula, we have

$$
\begin{gathered}
d \Phi(t)=\frac{\Phi(t)}{\rho^{2}+\left\|z^{\varepsilon}(\cdot, t)\right\|_{L_{2}(\mu)}^{2}}\left[\int_{\mathbb{R}} \int_{\mathbb{R}} \psi^{2}(t, u, p) \mu(d u) d p+\right. \\
\left.+\frac{1}{2}\left(\frac{1}{\varepsilon}-1\right) \frac{1}{\rho^{2}+\left\|z^{\varepsilon}(\cdot, t)\right\|_{L_{2}(\mu)}^{2}} \int_{\mathbb{R}}\left(\int_{\mathbb{R}} z^{\varepsilon}(u, t) \psi(t, u, p) \mu(d u)\right)^{2} d p\right] d t+ \\
+\frac{2}{\sqrt{\varepsilon}} \frac{\Phi(t)}{\rho^{2}+\left\|z^{\varepsilon}(\cdot, t)\right\|_{L_{2}(\mu)}^{2}} \int_{\mathbb{R}} \int_{\mathbb{R}} z^{\varepsilon}(u, t) \psi(t, u, p) \mu(d u) W(d p, d t)= \\
=\int_{\mathbb{R}} f_{1}(p, t) W(d p, d t)+f_{2}(t) d t .
\end{gathered}
$$

Note that for $t \leq \tau$

$$
\int_{\mathbb{R}}\left(\int_{\mathbb{R}} z^{\varepsilon}(u, t) \psi(t, u, p) \mu(d u)\right)^{2} d p \leq
$$




$$
\begin{gathered}
\leq\left\|z^{\varepsilon}(\cdot, t)\right\|_{L_{2}(\mu)} L\left(\left\|z^{\varepsilon}(\cdot, t)\right\|_{L_{2}(\mu)}+\rho^{2}\right) \leq \\
\leq L\left(\left\|z^{\varepsilon}(\cdot, t)\right\|_{L_{2}(\mu)}+\rho^{2}\right)^{2} .
\end{gathered}
$$

Then

$$
\int_{\mathbb{R}} f_{1}^{2}(p, t) d p \leq \frac{4 L \Phi^{2}(t)}{\varepsilon}
$$

and

$$
f_{2}(t) \leq \frac{L \Phi(t)}{\varepsilon}
$$

Fix $\delta>0$ and define the stopping time $\tau_{1}=\inf \left\{t:\left\|z^{\varepsilon}(\cdot, t)\right\|_{L_{2}(\mu)} \geq \delta\right\} \wedge \tau$. Since the norm $\left\|f_{1}(t, \cdot)\right\|_{L_{2}(\mathbb{R})} \leq \frac{2 \sqrt{L}}{\sqrt{\varepsilon}} \Phi(t)$ is uniformly bounded on $\left[0, \tau_{1}\right]$, it follows that $\left(\Phi(t)-\int_{0}^{t} f_{2}(s) d s\right)$ is a continuous martingale up to $\tau_{1}$. Therefore

$$
\begin{gathered}
E \Phi\left(t \wedge \tau_{1}\right)=\Phi(0)+E \int_{0}^{t \wedge \tau_{1}} f_{2}(s) d s \leq \\
\leq \Phi(0)+\frac{L}{\varepsilon} E \int_{0}^{t \wedge \tau_{1}} \Phi(s) d s=\Phi(0)+\frac{L}{\varepsilon} E \int_{0}^{t \wedge \tau_{1}} \Phi\left(s \wedge \tau_{1}\right) d s= \\
=\Phi(0)+\frac{L}{\varepsilon} \int_{0}^{t} E \Phi\left(s \wedge \tau_{1}\right) d s .
\end{gathered}
$$

Consequently, by Gronwall's lemma

$$
E \Phi\left(\tau_{1}\right)=E \Phi\left(\tau_{1} \wedge 1\right) \leq \Phi(0) e^{L / \varepsilon} .
$$

Therefore, by Chebychev's inequality, we have that

$$
\begin{gathered}
P\left\{\left\|z^{\varepsilon}\left(\cdot, \tau_{1}\right)\right\|_{L_{2}(\mu)} \geq \delta\right\}=P\left\{f\left(\left\|z^{\varepsilon}\left(\cdot, \tau_{1}\right)\right\|_{L_{2}(\mu)}\right) \geq f(\delta)\right\} \leq \\
\leq \frac{E f\left(\left\|z^{\varepsilon}\left(\cdot, \tau_{1}\right)\right\|_{L_{2}(\mu)}\right)}{f(\delta)}=\frac{E \Phi\left(\tau_{1}\right)}{f(\delta)} .
\end{gathered}
$$

Since $\sup _{t \in[0, \tau]}\left\|z^{\varepsilon}(\cdot, t)\right\|_{L_{2}(\mu)} \geq \delta$ iff $\left\|z^{\varepsilon}\left(\cdot, \tau_{1}\right)\right\|_{L_{2}(\mu)} \geq \delta$, then

$$
\begin{gathered}
\varepsilon \log P\left\{\sup _{t \in[0, \tau]}\left\|z^{\varepsilon}(\cdot, t)\right\|_{L_{2}(\mu)} \geq \delta\right\}=\varepsilon \log P\left\{\left\|z^{\varepsilon}\left(\cdot, \tau_{1}\right)\right\|_{L_{2}(\mu)} \geq \delta\right\} \leq \\
\leq \varepsilon \log \frac{\left(\rho^{2}+\|\alpha\|_{L_{2}(\mu)}^{2}\right)^{1 / \varepsilon} e^{L / \varepsilon}}{f(\delta)}=L+\log \frac{\rho^{2}+\|\alpha\|_{L_{2}(\mu)}^{2}}{\rho^{2}+\delta^{2}} .
\end{gathered}
$$

The lemma is proved. 
Lemma 2.3. For $\alpha<1 / 2$ there exists a constant $C>0$ such that for any $0<\varepsilon<1, m \geq 1$ :

$$
E \exp \left\{\alpha \sup _{t \in\left[\frac{i}{m}, \frac{i+1}{m}\right]} \frac{m}{\varepsilon}\left\|x_{m}^{\varepsilon}(\cdot, t)-x_{m}^{\varepsilon}\left(\cdot, \frac{i}{m}\right)\right\|_{L_{2}(\mu)}^{2}\right\} \leq C, i=0, \ldots, m-1 .
$$

Proof. Let us write

$$
\begin{aligned}
& \exp \left\{\alpha \sup _{t \in\left[\frac{i}{m}, \frac{i+1}{m}\right]} \frac{m}{\varepsilon}\left\|x_{m}^{\varepsilon}(\cdot, t)-x_{m}^{\varepsilon}\left(\cdot, \frac{i}{m}\right)\right\|_{L_{2}(\mu)}^{2}\right\}= \\
= & \sum_{n=0}^{\infty} \frac{\alpha^{n}}{n !}\left(\sup _{t \in\left[\frac{i}{m}, \frac{i+1}{m}\right]} \frac{m}{\varepsilon}\left\|x_{m}^{\varepsilon}(\cdot, t)-x_{m}^{\varepsilon}\left(, \frac{i}{m}\right)\right\|_{L_{2}(\mu)}^{2}\right)^{n} .
\end{aligned}
$$

A general term of this series can be estimated as

$$
\begin{gathered}
E\left(\sup _{t \in\left[\frac{i}{m}, \frac{i+1}{m}\right]}\left\|x_{m}^{\varepsilon}(\cdot, t)-x_{m}^{\varepsilon}\left(\cdot, \frac{i}{m}\right)\right\|_{L_{2}(\mu)}^{2}\right)^{n} \leq \\
\leq E \varepsilon^{n}\left(\int_{\mathbb{R}} \sup _{t \in\left[\frac{i}{m}, \frac{i+1}{m}\right]}\left(\int_{\frac{i}{m}}^{t} \int_{\mathbb{R}} \varphi\left(x_{m}^{\varepsilon}\left(u, \frac{i}{m}\right)-p\right) W(d p, d s)\right)^{2} \mu(d u)\right)^{n} \leq \\
\leq \varepsilon^{n} \int_{\mathbb{R}} E \sup _{t \in\left[\frac{i}{m}, \frac{i+1}{m}\right]}\left(\int_{\frac{i}{m}}^{t} \int_{\mathbb{R}} \varphi\left(x_{m}^{\varepsilon}\left(u, \frac{i}{m}\right)-p\right) W(d p, d s)\right)^{2 n} \mu(d u) .
\end{gathered}
$$

Since $\int_{\frac{i}{m}}^{t} \int_{\mathbb{R}} \varphi\left(x_{m}^{\varepsilon}\left(u, \frac{i}{m}\right)-p\right) W(d p, d s)$ is a continuous martingale, it follows that $[12]$

$$
\begin{gathered}
E\left(\sup _{t \in\left[\frac{i}{m}, \frac{i+1}{m}\right]}\left\|x_{m}^{\varepsilon}(\cdot, t)-x_{m}^{\varepsilon}\left(\cdot, \frac{i}{m}\right)\right\|_{L_{2}(\mu)}^{2}\right)^{n} \leq \\
\leq \varepsilon^{n} \int_{\mathbb{R}}\left(\frac{2 n}{2 n-1}\right)^{2 n} E\left(\int_{\frac{i}{m}}^{\frac{i+1}{m}} \int_{\mathbb{R}} \varphi\left(x_{m}^{\varepsilon}\left(u, \frac{i}{m}\right)-p\right) W(d p, d s)\right)^{2 n} \mu(d u)= \\
=\left(\frac{\varepsilon}{m}\right)^{n}\left(\frac{2 n}{2 n-1}\right)^{2 n}(2 n-1) ! !
\end{gathered}
$$

Consequently for $\alpha<1 / 2$ the constant $C$ can be chosen to be

$$
C=\sum_{n=0}^{\infty} \frac{\alpha^{n}}{n !}\left(\frac{2 n}{2 n-1}\right)^{2 n}(2 n-1) ! !
$$

The lemma is proved. 
The following lemma shows that $\left\{x_{m}^{\varepsilon}\right\}$ is exponentially good approximation of $\left\{x^{\varepsilon}\right\}$.

Lemma 2.4. For any $\delta>0$,

$$
\lim _{m \rightarrow \infty} \varlimsup_{\varepsilon \rightarrow 0} \varepsilon \log P\left\{\left\|x^{\varepsilon}-x_{m}^{\varepsilon}\right\|>\delta\right\}=-\infty .
$$

Proof. Fix $\delta>0$. For any $\rho>0$, define the stopping time

$$
\tau=\inf \left\{t \mid\left\|x_{m}^{\varepsilon}(\cdot, t)-x_{m}^{\varepsilon}\left(\cdot, \frac{[m t]}{m}\right)\right\|_{L_{2}(\mu)} \geq \rho\right\} \wedge 1 .
$$

Let $z^{\varepsilon}(u, t)=x^{\varepsilon}(u, t)-x_{m}^{\varepsilon}(u, t)$. Due to Lemma 2.2 for any $\delta>0$ and any $\varepsilon \leq 1$,

$$
\varepsilon \log P\left\{\sup _{t \in[0, \tau]}\left\|x^{\varepsilon}(\cdot, t)-x_{m}^{\varepsilon}(\cdot, t)\right\|_{L_{2}(\mu)}>\delta\right\} \leq L+\log \frac{\rho^{2}}{\rho^{2}+\delta^{2}},
$$

where $L$ is independent of $\varepsilon, \delta, \rho$ and $m$. Hence,

$$
\limsup _{\rho \rightarrow 0} \varlimsup_{m \geq 1} \varepsilon \lim \varepsilon \log P\left\{\sup _{t \in[0, \tau]}\left\|x^{\varepsilon}(\cdot, t)-x_{m}^{\varepsilon}(\cdot, t)\right\|_{L_{2}(\mu)}>\delta\right\}=-\infty .
$$

Now, since

$$
\begin{gathered}
\left\{\left\|x^{\varepsilon}-x_{m}^{\varepsilon}\right\|>\delta\right\}= \\
=\left\{\sup _{t \in[0, \tau]}\left\|x^{\varepsilon}(\cdot, t)-x_{m}^{\varepsilon}(\cdot, t)\right\|_{L_{2}(\mu)}>\delta\right\} \cup\left\{\sup _{t \in(\tau, 1]}\left\|x^{\varepsilon}(\cdot, t)-x_{m}^{\varepsilon}(\cdot, t)\right\|_{L_{2}(\mu)}>\delta\right\} \subset \\
\subset\left\{\sup _{t \in[0, \tau]}\left\|x^{\varepsilon}(\cdot, t)-x_{m}^{\varepsilon}(\cdot, t)\right\|_{L_{2}(\mu)}>\delta\right\} \cup\{\tau<1\},
\end{gathered}
$$

the lemma will be proved as soon as we show that for all $\rho>0$,

$$
\lim _{m \rightarrow \infty} \varlimsup_{\varepsilon \rightarrow 0} \varepsilon \log P\left\{\sup _{t \in[0 ; 1]}\left\|x_{m}^{\varepsilon}(\cdot, t)-x_{m}^{\varepsilon}\left(\cdot, \frac{[m t]}{m}\right)\right\|_{L_{2}(\mu)} \geq \rho\right\}=-\infty .
$$

It follows from Chebychev's inequality and Lemma 2.3 that

$$
\begin{gathered}
P\left\{\sup _{t \in[0 ; 1]}\left\|x_{m}^{\varepsilon}(\cdot, t)-x_{m}^{\varepsilon}\left(\cdot, \frac{[m t]}{m}\right)\right\|_{L_{2}(\mu)} \geq \rho\right\} \leq \\
\leq m \max _{0 \leq i \leq m-1} P\left\{\sup _{t \in\left[\frac{i}{m}, \frac{i+1}{m}\right]} \frac{m}{\varepsilon}\left\|x_{m}^{\varepsilon}(\cdot, t)-x_{m}^{\varepsilon}\left(\cdot, \frac{i}{m}\right)\right\|_{L_{2}(\mu)}^{2} \geq \frac{m \rho^{2}}{\varepsilon}\right\} \leq m C e^{-\frac{m \rho^{2}}{\varepsilon}} .
\end{gathered}
$$

The lemma is proved. 
Lemma 2.1 and Lemma 2.4 give us possibility to receive the LDP for $\left\{x^{\varepsilon}\right\}$ using the following theorem from [9].

Theorem 2.2. Let $(\mathfrak{X}, \rho),(\mathcal{Y}, \sigma)$ be Polish spaces, $\left\{y^{\varepsilon}\right\}$ satisfies the LDP with rate function $I, G_{m}: \mathcal{Y} \rightarrow \mathfrak{X}, m \geq 1$, are continuous functions. Assume that there exists $G: \mathcal{Y} \rightarrow \mathfrak{X}$ such that for every $\alpha<\infty$,

$$
\lim _{m \rightarrow \infty} \sup _{\{y: I(y) \leq \alpha\}} \rho\left(G_{m}(y), G(y)\right)=0 .
$$

Then any family $\left\{x^{\varepsilon}\right\}$ for which $\left\{G_{m}\left(y^{\varepsilon}\right)\right\}$ is exponentially good approximation satisfies the LDP in $\mathfrak{X}$ with rate function

$$
I^{\prime}(x)=\inf \{I(y): x=G(y)\} .
$$

Define

$$
\begin{gathered}
H_{1}=\left\{h \in C\left([0 ; 1] ; L_{2}(\mu)\right): h(u, t)=\right. \\
=u+\int_{0}^{t} \int_{\mathbb{R}} \varphi(h(u, s)-p) a(p, s) d p d s, a \in L_{2}(\mathbb{R} \times[0 ; 1]\} .
\end{gathered}
$$

Theorem 2.3. The family $\left\{x^{\varepsilon}\right\}$ satisfies the LDP in $\mathfrak{X}$ with rate function

$$
I(h)=\left\{\begin{array}{l}
\frac{1}{4 \pi} \int_{0}^{1} \int_{\mathbb{R}}\left(\frac{\mathcal{F}\left(\dot{h}\left(h^{-1}(\cdot, s), s\right)\right)(\lambda)}{\mathcal{F}(\varphi)(\lambda)}\right)^{2} d \lambda d s, h \in H_{1}, \\
\infty, h \notin H_{1} .
\end{array}\right.
$$

Proof. Define the map $G_{m}$ as $h=G_{m}(g), g \in \mathcal{Y}$, where

$$
\begin{gathered}
h(u, t)=h\left(u, \frac{k}{m}\right)+\int_{\mathbb{R}} \psi_{1}\left(h\left(u, \frac{k}{m}\right)-q\right)\left[g(q, t)-g\left(q, \frac{k}{m}\right)\right] d q, \\
t \in\left[\frac{k}{m}, \frac{k+1}{m}\right], k=0,1, \ldots, m-1, \\
h(0, u)=u .
\end{gathered}
$$

Now, observe that $G_{m}$ is continuous by Lemma 2.1 and that $x_{m}^{\varepsilon}=G_{m}\left(y^{\varepsilon}\right)$ where $y^{\varepsilon}$ was defined in (2.9). It is enough to define $G$ on $H_{2}=\left\{h \in C\left([0 ; 1] ; L_{2}(\mu)\right): h(u, t)=u+\int_{0}^{t} \int_{\mathbb{R}} \psi_{2}(u-p) a(p, s) d p d s\right\}$.

Let $f=G(g)$ be the unique solution of the integral equation

$$
f(t, u)=u+\int_{0}^{t} \int_{\mathbb{R}} \psi_{1}(f(u, s)-q) \dot{g}(q, s) d q d s .
$$


In view of Lemma 2.4 the proof of the theorem is completed by combining Theorem 2.1 and Theorem 2.2, as soon as we show that for every $\alpha<\infty$,

$$
\lim _{m \rightarrow \infty} \sup _{\left\{g \mid I_{y}(g) \leq \alpha\right\}}\left\|G_{m}(g)-G(g)\right\|=0 .
$$

To this end, fix $\alpha<\infty$ and $g \in H_{2}$ such that $I_{y}(g) \leq \alpha$. For $g \in H_{2}$, there exists $a \in L_{2}(\mathbb{R} \times[0,1])$ such that

$$
\dot{g}(u, t)=\int_{\mathbb{R}} \psi_{2}(u-p) a(p, t) d p .
$$

Since $\varphi=\psi_{1} * \psi_{2}$ and $\|\varphi\|_{L_{2}(\mathbb{R})}=1$, it follows from the Cauchy-Schwarz inequality that for all $t \in[0,1]$

$$
\begin{gathered}
\left\|h(\cdot, t)-h\left(\cdot, \frac{[t m]}{m}\right)\right\|_{L_{2}(\mu)}^{2}= \\
=\int_{\mathbb{R}}\left(\int_{\frac{[t m]}{m}}^{t} \int_{\mathbb{R}} \varphi\left(h\left(u, \frac{[t m]}{m}\right)-p\right) a(p, s) d p d s\right)^{2} \mu(d u) \leq \\
\leq \frac{1}{m} \int_{\mathbb{R}} \int_{\frac{[t m]}{m}}^{t} \int_{\mathbb{R}} \varphi^{2}\left(h\left(u, \frac{[t m]}{m}\right)-p\right) d p \int_{\mathbb{R}} a^{2}(p, s) d p d s \mu(d u)= \\
=\frac{1}{m} \int_{\frac{[t m]}{m}}^{t} \int_{\mathbb{R}} a^{2}(p, s) d p d s \leq \frac{2 \alpha}{m} .
\end{gathered}
$$

The Cauchy-Schwarz inequality and the Lipschitz continuity of $\varphi$ imply that for all $t \in[0 ; 1]$ we have

$$
\begin{gathered}
\|h(\cdot, t)-f(\cdot, t)\|_{L_{2}(\mu)}^{2}= \\
=\int_{\mathbb{R}}\left(\int_{0}^{t} \int_{\mathbb{R}}\left[\varphi\left(h\left(u, \frac{[s m]}{m}\right)-p\right)-\varphi(f(u, s)-p)\right] a(p, s) d p d s\right)^{2} \mu(d u) \leq \\
\leq t L \int_{0}^{t} \int_{\mathbb{R}} a^{2}(p, s) d p\left\|f(\cdot, s)-h\left(\cdot, \frac{[s m]}{m}\right)\right\|_{L_{2}(\mu)}^{2} d s \leq \\
\leq t L \int_{0}^{t} \int_{\mathbb{R}} a^{2}(p, s) d p\left[\|f(\cdot, s)-h(\cdot, s)\|_{L_{2}(\mu)}^{2}+\frac{2 \alpha}{m}\right] d s \leq \\
\leq \frac{4 L \alpha^{2}}{m}+L \int_{0}^{t} \int_{\mathbb{R}} a^{2}(p, s) d p\|f(\cdot, s)-h(\cdot, s)\|_{L_{2}(\mu)}^{2} d s .
\end{gathered}
$$


Hence, by Gronwall's lemma,

$$
\|f(\cdot, t)-h(\cdot, t)\|_{L_{2}(\mu)}^{2} \leq \frac{4 L \alpha^{2}}{m} e^{L \int_{0}^{t} \int_{\mathbb{R}} a^{2}(p, s) d p d s} \leq \frac{4 L \alpha^{2}}{m} e^{2 L \alpha},
$$

which establishes (2.10) and proves the theorem.

Remark. Note that the distribution of $x^{\varepsilon}$ can be obtained not only by the phase scaling but by time changing also. Namely, denote $\widetilde{x}^{\varepsilon}(u, t)=x(u, \varepsilon t)$, $u \in \mathbb{R}, t \in[0 ; 1]$, where $x$ is defined by (1.1).

Lemma 2.5. $x^{\varepsilon}$ is equal in distribution to $\widetilde{x}^{\varepsilon}$.

Proof. Consider the $n$-point motions of both flows: $\left(x^{\varepsilon}\left(u_{1}, \cdot\right), x^{\varepsilon}\left(u_{2}, \cdot\right), \ldots\right.$, $\left.x^{\varepsilon}\left(u_{n}, \cdot\right)\right)$ and $\left(\widetilde{x}^{\varepsilon}\left(u_{1}, \cdot\right), \widetilde{x}^{\varepsilon}\left(u_{2}, \cdot\right), \ldots, \widetilde{x}^{\varepsilon}\left(u_{n}, \cdot\right)\right)$. They are diffusion processes in $\mathbb{R}$ with zero drift and diffusion matrices $A=\left(a_{i j}\right)_{i, j=1}^{n}$ and $\widetilde{A}=\left(\widetilde{a}_{i j}\right)_{i, j=1}^{n}$.

For the first process we have

$$
\begin{gathered}
a_{i j}(\bar{u})= \\
=\lim _{t \rightarrow 0} \frac{1}{t} E_{\bar{u}} \varepsilon \int_{0}^{t} \int_{\mathbb{R}} \varphi\left(x^{\varepsilon}\left(u_{i}, s\right)-p\right) W(d p, d s) \int_{0}^{t} \int_{\mathbb{R}} \varphi\left(x^{\varepsilon}\left(u_{j}, s\right)-p\right) W(d p, d s)= \\
=\varepsilon \lim _{t \rightarrow 0} \frac{1}{t} \int_{0}^{t} \int_{\mathbb{R}} E_{\bar{u}} \varphi\left(x^{\varepsilon}\left(u_{i}, s\right)-p\right) \varphi\left(x^{\varepsilon}\left(u_{j}, s\right)-p\right) d p d s= \\
=\varepsilon \int_{\mathbb{R}} \varphi\left(u_{i}-p\right) \varphi\left(u_{j}-p\right) d p, \bar{u}=\left(u_{1}, \ldots, u_{n}\right) \in \mathbb{R}^{n} .
\end{gathered}
$$

For the second one we can write

$$
\begin{gathered}
\widetilde{a}_{i j}(\bar{u})= \\
=\lim _{t \rightarrow 0} \frac{1}{t} E_{\bar{u}} \int_{0}^{\varepsilon t} \int_{\mathbb{R}} \varphi\left(x\left(u_{i}, s\right)-p\right) W(d p, d s) \int_{0}^{\varepsilon t} \int_{\mathbb{R}} \varphi\left(x\left(u_{j}, s\right)-p\right) W(d p, d s)= \\
=\varepsilon \lim _{t \rightarrow 0} \frac{1}{t} \int_{0}^{t} \int_{\mathbb{R}} E_{\bar{u}} \varphi\left(x\left(u_{i}, \varepsilon s\right)-p\right) \varphi\left(x\left(u_{j}, \varepsilon s\right)-p\right) d p d s= \\
=\varepsilon \int_{\mathbb{R}} \varphi\left(u_{i}-p\right) \varphi\left(u_{j}-p\right) d p, \bar{u}=\left(u_{1}, \ldots, u_{n}\right) \in \mathbb{R}^{n} .
\end{gathered}
$$

Thus, the $n$-point motions of $x^{\varepsilon}$ and $\widetilde{x}^{\varepsilon}$ are equal in distribution. Therefore $x^{\varepsilon}$ is equal in distribution to $\widetilde{x}^{\varepsilon}$. 


\section{Arratia's flow and related stochastic calculus}

This section is devoted to Arratia's flow of coalescing Brownian particles. As it was mentioned in the first section, Arratia's flow can be considered as limit case of flows with the smooth correlation. It consists of Brownian particles, which move independently up to the meeting then stick and move together. In contrast with the smooth correlation case Arratia's flow can not be described by any stochastic differential equation with a Gaussian noise. So the LDP for Arratia's flow should be described using its intrinsic properties. In this section we present some facts about Arratia's flow, which will be useful later on. Arratia's flow can be defined by different ways $[1,2,5]$. We will use the following definition.

Definition 3.1. Arratia's flow $\{x(u, t) ; u \in \mathbb{R}, t \in[0 ; 1]\}$ is a random field with the properties:

1) for every $u \in \mathbb{R}\{x(u, t), t \in[0 ; 1]\}$ is a Wiener martingale with respect to a common filtration and $x(u, 0)=u$,

2) for every $u_{1} \leq u_{2}, t \in[0 ; 1]$

$$
x\left(u_{1}, t\right) \leq x\left(u_{2}, t\right),
$$

3) $\left\langle x\left(u_{1}, \cdot\right), x\left(u_{2}, \cdot\right)\right\rangle_{t}=(t-\tau), t>\tau, \tau=\inf \left\{s: x\left(u_{1}, s\right)=x\left(u_{2}, s\right)\right\}$.

It was proved in [13], that $x$ has a modification, which is a càdlàg Markov process in $C([0 ; 1])(u \in \mathbb{R}$ now plays the role of time). Further we will consider such modification.

We will establish the LDP for the family of random flows $\left\{x^{\varepsilon} ; \varepsilon>0\right\}$, which is built from $x$ using the time-change. Define for $\varepsilon \in(0 ; 1]$

$$
x^{\varepsilon}(u, t)=x(u, \varepsilon t), u \in \mathbb{R}, t \in[0 ; 1] .
$$

To describe rate function we need the fundamental fact about Arratia's flow. This property can be formulated as follows.

Lemma 3.1. $[1,2,14]$. For every interval $[a ; b]$ and positive time $t$ the set $\{x(u, t) ; u \in[a ; b]\}$ is finite with probability one.

In [14] this fact was obtained as a consequence of a more general statement about the finiteness of the total time of free motion of particles in Arratia's 
flow. Consider a partition $\lambda$ of the interval $[a ; b]: a=u_{0}<\ldots<u_{n}=b$. As usual denote $|\lambda|=\max _{k=0, \ldots, n-1} u_{k+1}-u_{k}$. For $k=1, \ldots, n$ define the random time

$$
\tau\left(u_{k}\right)=\inf \left\{t: x\left(u_{k}, t\right)=x\left(u_{k-1}, t\right)\right\} \wedge 1 .
$$

For $k=0$ put $\tau\left(u_{0}\right)=1$. The following statement was proved in [14, 15].

Theorem 3.1. [14, 15]. There exists a random variable

$$
\Gamma=\sup _{\lambda} \sum_{k=0}^{n} \tau\left(u_{k}\right) .
$$

Here supremum means, that for arbitrary $\lambda$

$$
\Gamma \geq \sum_{k=0}^{n} \tau\left(u_{k}\right)
$$

and for arbitrary random variable $\zeta$ with property (3.1) the following inequality holds

$$
\Gamma \leq \zeta
$$

Moreover, $\Gamma$ can be obtained as a limit a.s.

$$
\Gamma=\lim _{|\lambda| \rightarrow 0} \sum_{k=0}^{n} \tau\left(u_{k}\right) .
$$

Using Theorem 3.1 the following stochastic integrals were built for a bounded measurable function $\varphi$

$$
\begin{aligned}
& \int_{a}^{b} \int_{0}^{\tau(u)} \varphi(x(u, s)) d s=P-\lim _{|\lambda| \rightarrow 0} \sum_{k=0}^{n} \int_{0}^{\tau\left(u_{k}\right)} \varphi(x(u, s)) d s \\
& \int_{a}^{b} \int_{0}^{\tau(u)} \varphi(x(u, s)) d x(u, s)=L_{2^{-}} \lim _{|\lambda| \rightarrow 0} \sum_{k=0}^{n} \int_{0}^{\tau\left(u_{k}\right)} \varphi(x(u, s)) d x(u, s) .
\end{aligned}
$$

Note that the left-hand side in (3.2) contains two symbols of integral and only one symbol of differential. It emphasizes that the second differential can be substituted by $\tau(u)$ which formally possesses the property

$$
\sum_{u \in[a, b]} \tau(u)<+\infty
$$


The integrals from (3.2) allow to formulate the Girsanov theorem for Arratia's flow [14]. If we consider the flow $y$, which is built analogously to Arratia's flow but instead of a Wiener process a diffusion process with the drift $\varphi$ and variance 1 is used, then it can be proved [14], that in an appropriate functional space the distribution $P_{y}$ of this flow is absolutely continuous with respect to the distribution $P_{x}$ of Arratia's flow and

$$
\frac{d P_{y}}{d P_{x}}=\exp \left\{\int_{a}^{b} \int_{0}^{\tau(u)} \varphi(x(u, s)) d x(u, s)-\frac{1}{2} \int_{a}^{b} \int_{0}^{\tau(u)} \varphi^{2}(x(u, s)) d s\right\} .
$$

Note that the form of the derivative in (3.3) is very natural. It consists of the sum of usual terms for Girsanov theorem along the pieces of trajectories of particles in Arratia's flow up to the moment of the first meeting. We will prove, that rate function for $\left\{x^{\varepsilon}\right\}$ is the infinite sum of rate functions for the Wiener process $x(u, \cdot)-u, u \in \mathbb{R}$ up to the moment of the meeting. The main result will be proved in two steps. In the next two sections we will consider the case of finite number of particles and the general case will be treated in the last section.

\section{LDP for stopped Wiener process}

Here we consider a Wiener process $\vec{w}$ in $\mathbb{R}^{d}$ starting from a point $\vec{u}$. Let $B \subset \mathbb{R}^{d}$ be a closed set. Define the stopping time

$$
\tau=\inf \{t: \vec{w}(t) \in B\} \wedge 1 .
$$

Consider in the space $C\left([0 ; 1], \mathbb{R}^{d}\right)$ the family of random elements $\left\{\vec{y}^{\varepsilon} ; \varepsilon \in\right.$ $(0 ; 1]\}$ defined as follows

$$
\vec{y}^{\varepsilon}(t)=\vec{\eta}(\varepsilon t), t \in[0 ; 1],
$$

where $\vec{\eta}(t)=\vec{w}(t \wedge \tau)$. To describe rate function for the family $\left\{\vec{y}^{\varepsilon}\right\}$ let us denote for $\vec{f} \in C\left([0 ; 1], \mathbb{R}^{d}\right)$

$$
\tau(\vec{f})=\inf \{t: \vec{f}(t) \in B\} \wedge 1 .
$$

Define

$$
\Phi(\vec{f})(t)=\vec{f}(t \wedge \tau(\vec{f})), t \in[0 ; 1]
$$


Denote by $H$ the subset of $C\left([0 ; 1], \mathbb{R}^{d}\right)$ consisting of functions with squareintegrable derivative. Now define the rate function $I$ on $C\left([0 ; 1], \mathbb{R}^{d}\right)$ as follows

$$
I(\vec{g})=\left\{\begin{array}{l}
\frac{1}{2} \int_{0}^{1}\|\dot{\vec{g}}(t)\|^{2} d t, \vec{g}(0)=\vec{u}, \vec{g} \in H \cap \Phi\left(C\left([0 ; 1], \mathbb{R}^{d}\right)\right), \\
+\infty, \vec{g}(0) \neq \vec{u} \text { or } \vec{g} \notin H \cap \Phi\left(C\left([0 ; 1], \mathbb{R}^{d}\right)\right) .
\end{array}\right.
$$

The following statement is the main result of this section.

Theorem 4.1. The family $\left\{\vec{y}^{\varepsilon}\right\}$ satisfies the $L D P$ in $C\left([0 ; 1], \mathbb{R}^{d}\right)$ with rate function $I$.

Before proving the theorem let us note that it can not be obtained directly from the LDP for Wiener process by contraction principle or its modification [9], since the map $\Phi$ is not continuous. Moreover, in some cases the set of discontinuities for $\Phi$ has a positive Wiener measure. The following example shows such possibility.

Example 4.1. Let $d=2$ and $B$ be a Sierpinski carpet of the positive Lebesgue measure, which is built by the usual procedure [16],

$$
B=\cap_{n=1}^{\infty} B_{n} .
$$

Here for every $n \geq 1 \quad B_{n}$ is a finite union of disjoint closed squares, $B_{1}=$ $[0 ; 1]^{2}, B_{n+1} \subset B_{n}^{\circ}, n \geq 1$. The squares in $B_{n}$ have equal sides $d_{n}$ and $d_{n} \rightarrow$ $0, n \rightarrow \infty$. By the Kakutany criterium the process $\vec{w}$ visits $B$ with a positive probability, i.e.

$$
P\{\exists t \in[0 ; 1): \vec{w}(t) \in B\}>0 .
$$

Now consider the subset $A \subset C\left([0 ; 1], \mathbb{R}^{d}\right)$ consisting of functions with the properties

$$
\tau(\vec{f})<1, \Phi(\vec{f}) \neq \vec{f} .
$$

It can be proved, that $A$ is a Borel set. It follows from (4.1), that $A$ has a positive Wiener measure. Now check, that every point of $A$ is a point of discontinuity for $\Phi$. Let $\vec{f} \in A$. For every $n \geq 1$ define $\Delta_{n}=\{t \in[0 ; 1]: \vec{f}(t) \in$ $\left.B_{n}^{\circ}\right\}$. Then $\Delta_{n}$ is a union of disjoint open intervals (possibly with one interval of the type $(\alpha ; 1])$. Consider one interval $(\alpha ; \beta)$ from $\Delta_{n}$. Then $\vec{f}([\alpha ; \beta])$ is a subset of one of the squares which form $B_{n}$. Denote this square by $J$. Then $\vec{f}(\alpha), \vec{f}(\beta) \in \partial J$. Define the function $\vec{f}_{n}$ on $[\alpha ; \beta]$ in such a way that it is continuous and $\vec{f}_{n}([\alpha ; \beta]) \subset \partial J, \vec{f}_{n}(\alpha)=\vec{f}(\alpha), \vec{f}_{n}(\beta)=\vec{f}(\beta)$. If we proceed in 
the same way on every interval from $\Delta_{n}$ and define for $t \notin \Delta_{n} \vec{f}_{n}(t)=\vec{f}(t)$, then we will get $\vec{f}_{n} \in C\left([0 ; 1], \mathbb{R}^{2}\right)$. This function has the following properties:

1) $\max _{t \in[0 ; 1]}\left\|\vec{f}_{n}(t)-\vec{f}(t)\right\| \leq 2 d_{n} \rightarrow 0, n \rightarrow \infty$,

2) $\tau\left(\vec{f}_{n}\right)=1, n \geq 1$.

Consequently,

$$
\Phi\left(\vec{f}_{n}\right)=\vec{f}_{n}, n \geq 1
$$

and

$$
\Phi\left(\overrightarrow{f_{n}}\right) \rightarrow \vec{f}, n \rightarrow \infty .
$$

But $\vec{f} \neq \Phi(\vec{f})$. Hence $\vec{f}$ is a point of discontinuity for $\Phi$. Note that the crucial point in this example is the possibility to approximate uniformly arbitrary Wiener trajectory by continuous functions, that do not take values in the set $B$.

Proof of Theorem 4.1. Let $G$ be an open set in $C\left([0 ; 1], \mathbb{R}^{d}\right)$. Denote by $I_{0}$ the rate function for the family

$$
\vec{w}^{\varepsilon}(t)=\vec{w}(\varepsilon t), t \in[0 ; 1], \varepsilon \in(0 ; 1],
$$

i.e.

$$
I_{0}(\vec{g})= \begin{cases}\frac{1}{2} \int_{0}^{1}\|\dot{\vec{g}}(t)\|^{2} d t, & \vec{g}(0)=\vec{u}, \vec{g} \in H, \\ +\infty, & \vec{g}(0) \neq \vec{u} \text { or } \vec{g} \notin H .\end{cases}
$$

Note that

$$
\vec{y}^{\varepsilon}=\Phi\left(\vec{w}_{\varepsilon}\right), \varepsilon \in(0 ; 1] .
$$

We will prove, that

$$
\inf _{G} I= \begin{cases}\inf _{\Phi^{-1}(G)^{\circ}} I_{0}, & \Phi^{-1}(G)^{\circ} \neq \varnothing, \\ +\infty, & \Phi^{-1}(G)^{\circ}=\varnothing .\end{cases}
$$

Here, as usual, $A^{\circ}$ denote the interior of the set $A$. Consider first the case when $\Phi^{-1}(G)^{\circ}=\varnothing$. Let $\vec{f} \in G$. Note that if $\tau(\vec{f})=1$, then there exists $\vec{g} \in G$ close enough to $\vec{f}$ such that $\vec{g}([0 ; 1]) \cap B=\varnothing$. The function $\vec{g}$ can be defined as

$$
\vec{g}(t)=\vec{f}(t \wedge 1-\delta)
$$

for small enough $\delta$. Then there exists $\sigma>0$ such, that the open ball $B(\vec{g}, \sigma)$ with the center $\vec{g}$ and radius $\sigma$ is subset of $G$ and has the property

$$
\forall \vec{h} \in B(\vec{g}, \sigma): \vec{h}([0 ; 1]) \cap B=\varnothing .
$$


Hence

$$
\forall \vec{h} \in B(\vec{g}, \sigma): \Phi(\vec{h})=\vec{h} .
$$

Consequently, $B(\vec{g}, \sigma) \subset \Phi^{-1}(G)$ which contradicts our supposition. It follows from the previous considerations, that now

$$
\forall \vec{f} \in G: \tau(\vec{f})<1 .
$$

Consider $\vec{f} \in G$ such, that

$$
\Phi^{-1}(\vec{f})=\varnothing .
$$

For such $\vec{f}$ by definition $I(\vec{f})=+\infty$. Now let $\Phi^{-1}(\vec{f}) \neq \varnothing$. Then $\vec{f}=\Phi(\vec{f})$. Note that the open set $G$ contains with the function $\vec{f}$ every function of the kind

$$
\vec{h}(t)=\vec{f}(t \wedge \tau(\vec{f})-\delta)
$$

for small enough $\delta$. But for such $\vec{h} \tau(\vec{h})=1$ which again contradicts our supposition $\Phi^{-1}(G)^{\circ}=\varnothing$.

It remains to consider the case $\Phi^{-1}(G)^{\circ} \neq \varnothing$. Let $\vec{f}$ belongs to $G$. If $\tau(f)=$ 1 and $I(f)<+\infty$, then, similarly to the previous considerations, there exists a sequence $\left\{\vec{h}_{n} ; n \geq 1\right\}$ from $\Phi^{-1}(G)^{\circ}$ such, that $\vec{h}_{n} \rightarrow \vec{f}, I\left(\vec{h}_{n}\right) \rightarrow I(\vec{f})$, $n \rightarrow \infty$, and

$$
\forall n \geq 1: \vec{h}_{n}([0 ; 1]) \cap B=\varnothing .
$$

Since for such functions

$$
\begin{gathered}
I\left(\vec{h}_{n}\right)=I_{0}\left(\vec{h}_{n}\right), \\
\Phi\left(\vec{h}_{n}\right)=\vec{h}_{n},
\end{gathered}
$$

then

$$
I(\vec{f}) \geq \inf _{\Phi^{-1}(G)^{\circ}} I_{0} .
$$

Now take $\vec{f} \in G$ such, that $\tau(\vec{f})<1$ and $\Phi^{-1}(\vec{f})=\varnothing, I(\vec{f})<+\infty$. Using the approximation like (4.3) we again can get inequality (4.4). This completes the proof of relation (4.2).

Now for open set $G$

$$
\begin{gathered}
\varliminf_{\varepsilon \rightarrow 0} \varepsilon \log P\left\{y_{\varepsilon} \in G\right\}=\varliminf_{\varepsilon \rightarrow 0} \varepsilon \log P\left\{\Phi\left(w_{\varepsilon}\right) \in G\right\}= \\
=\varliminf_{\varepsilon \rightarrow 0} \varepsilon \log P\left\{w_{\varepsilon} \in \Phi^{-1}(G)\right\} \geq \varliminf_{\varepsilon \rightarrow 0} \varepsilon \log P\left\{w_{\varepsilon} \in \Phi^{-1}(G)^{0}\right\} \geq
\end{gathered}
$$




$$
\geq \inf _{\Phi^{-1}(G)^{\circ}} I_{0}=\inf _{G} I .
$$

Here we use the LDP for Wiener process.

Consider the closed set $F \subset C\left([0 ; 1], \mathbb{R}^{d}\right)$. Let us prove for $F$ an analog of (4.2). Namely,

$$
\inf _{F} I=\frac{\inf }{\Phi^{-1}(F)} I_{0},
$$

where $\bar{A}$ denote the closure of $A$. To check (4.5) take a function $\vec{f} \in \overline{\Phi^{-1}(F)}$. Suppose, that $I_{0}(\vec{f})<+\infty$.

If $\tau(\vec{f})=1$, then $\Phi(\vec{f})=\vec{f}$. From other side

$$
\vec{f}=\lim _{n \rightarrow \infty} f_{n}
$$

where

$$
\overrightarrow{f_{n}} \in \Phi^{-1}(F), n \geq 1
$$

Consequently,

$$
\begin{gathered}
\tau\left(\vec{f}_{n}\right) \rightarrow 1, n \rightarrow \infty, \\
\Phi\left(\vec{f}_{n}\right) \rightarrow \vec{f}, n \rightarrow \infty .
\end{gathered}
$$

Hence $\vec{f} \in \Phi^{-1}(F)$ and by definition $I_{0}(\vec{f})=I(\Phi(f))$.

Now, let $\tau(\vec{f})<1$. Then condition $I(\vec{f})<+\infty$ implies that $\Phi(\vec{f})=\vec{f}$. Consider a sequence $\left\{\vec{f}_{n} ; n \geq 1\right\}$ from $\Phi^{-1}(F)$ such, that

$$
\overrightarrow{f_{n}} \rightarrow \vec{f}, n \rightarrow \infty
$$

Then the sequence $\left\{\Phi\left(\vec{f}_{n}\right) ; n \geq 1\right\}$ contains subsequence converging to a certain element $\vec{g}$ of $F$. It can be easily verified, that $\vec{f}=\vec{g}$. Hence $\vec{f} \in F$ and $\vec{f} \in \Phi^{-1}(F)$. This proves (4.5). Now

$$
\begin{aligned}
& \varlimsup_{\varepsilon \rightarrow 0+} \varepsilon \log P\left\{\vec{y}^{\varepsilon} \in F\right\}=\varlimsup_{\varepsilon \rightarrow 0+} \varepsilon \log P\left\{\vec{w}^{\varepsilon} \in \Phi^{-1}(F)\right\} \leq \\
& \leq \varlimsup_{\varepsilon \rightarrow 0+} \varepsilon \log P\left\{\vec{w}^{\varepsilon} \in \overline{\Phi^{-1}(F)}\right\} \leq-\inf _{\Phi^{-1}(F)} I_{0}=-\inf _{F} I .
\end{aligned}
$$

The theorem is proved. 


\section{$5 \quad$ LDP for finite-dimensional distributions of Arratia's flow}

In this section we will consider the LDP for process $\vec{x}^{\varepsilon}=\left\{x\left(u_{1}, \varepsilon t\right), \ldots\right.$, $\left.x\left(u_{n}, \varepsilon t\right), t \in[0 ; 1]\right\}, \varepsilon \in(0 ; 1]$ where $x$ is Arratia's flow, $u_{1}<\ldots<u_{n}$ are fixed points. Denote by $C_{\vec{u}}\left([0 ; 1], \mathbb{R}^{n}\right)$ the subset of $C\left([0 ; 1], \mathbb{R}^{n}\right)$ consisting of functions with the property $f_{i}(0)=u_{i}, i=1, \ldots, n$. Consider the moments of sticking $\tau_{1} \leq \ldots \leq \tau_{n-1}$ (possibly from a certain number they are equal to $1)$. Using these moments one can define the map $\Phi$ on $C_{\vec{u}}\left([0 ; 1], \mathbb{R}^{n}\right)$ similarly as it was done in the previous section. Namely, after the moment of meeting of some coordinates put all of them to be equal to the coordinate with the smallest number. Then $\vec{x}=\Phi(\vec{w})$, where $\vec{w}$ is a standard Wiener process starting from $\left(u_{1}, \ldots, u_{n}\right)$. Exactly as in the previous section one can prove the following theorem.

Theorem 5.1. $\left\{\vec{x}^{\varepsilon}\right\}$ satisfies the LDP with rate function $I$. Here $I(\vec{f})$ is equal to $+\infty$ if $\Phi(\vec{f}) \neq \vec{f}$ or if $\vec{f}$ has not a square integrable derivative. In the opposite case

$$
I(\vec{f})=\frac{1}{2} \sum_{k=0}^{n} \int_{0}^{\tau\left(u_{k}\right)} f_{k}^{\prime}(s)^{2} d s .
$$

Here, as in section 3 ,

$$
\begin{aligned}
& \tau\left(u_{0}\right)=1, \\
& \tau\left(u_{k}\right)=\inf \left\{t: f_{k}(t)=f_{k-1}(t)\right\} \wedge 1, k=1, \ldots, n .
\end{aligned}
$$

\section{$6 \quad$ LDP for Arratia's flow on finite interval}

Consider Arratia's flow $\{x(u, t) ; u \in[0 ; 1], t \in[0 ; 1]\}$. Following [14] we suppose, that $x$ is already modified to be a càdlàg process with respect to the variable $u$ with values in $C([0 ; 1])$. Denote by $\lambda$ the Lebesgue measure on $[0 ; 1]$ and for every $t \in[0 ; 1]$ define the random measure $\mu_{t}$ as the image

$$
\mu_{t}=\lambda \circ x(\cdot, t)^{-1} .
$$

As it was proved in $[2,14], \mu_{t}$ for every $t>0$ is a random measure concentrated in a finite number of points. Note that $x$ can be fully recovered 
from $\left\{\mu_{t} ; t \in(0 ; 1]\right\}$. For probability measures on $\mathbb{R}$ we will use the LévyProkhorov distance $\sigma$ [17], which metrizes weak convergence. For $\varepsilon \in(0 ; 1]$ define

$$
\mu_{t}^{\varepsilon}=\mu_{\varepsilon t}, t \in[0 ; 1]
$$

We will establish the LDP for the processes $\mu^{\varepsilon}$. To do this consider the sequence of the $\left(2^{n}+1\right)$-point motions from Arratia's flow. For every $n \geq 1$ define $x_{n \varepsilon}$ as the family $\left\{x\left(\frac{k}{2^{n}}, \varepsilon t\right) ; k=0, \ldots, 2^{n}, t \in[0 ; 1]\right\}$. Recall $x_{n \varepsilon}$ satisfies the LDP in $C\left([0 ; 1], \mathbb{R}^{2^{n}+1}\right)$ accordingly to the previous section. Let us consider the sequence $\left\{x_{n \varepsilon} ; n \geq 1\right\}$ as an element of the product $\prod_{n=1}^{\infty} C([0 ; 1]$, $\mathbb{R}^{2^{n}+1}$ ) which is equipped with the product topology. Note that for every $k \leq n \quad x_{k \varepsilon}$ is a continuous function of $x_{n \varepsilon}$. Consequently, by the contraction principle, the vector $\left(x_{1 \varepsilon}, \ldots, x_{n \varepsilon}\right)$ satisfies the LDP in $\prod_{k=1}^{n} C\left([0 ; 1], \mathbb{R}^{2^{k}+1}\right)$ with rate function $I_{n}$ (the same as for $x_{n \varepsilon}$ ). Applying Dawson and Gärtner theorem about random sequences [18] one can get that $\left\{x_{n \varepsilon} ; n \geq 1\right\}$ satisfies the LDP in $\prod_{n=1}^{\infty} C\left([0 ; 1], \mathbb{R}^{2^{n}+1}\right)$ with rate function

$$
I\left(\left\{f_{n} ; n \geq 1\right\}\right)=\sup _{n \geq 1} I_{n}\left(f_{n}\right) .
$$

The last expression can be rewritten as follows. Consider a function $f$ which is defined on the set $\mathbb{Q}_{2}=\left\{\frac{k}{2^{n}} ; k=0, \ldots, 2^{n}, n \geq 1\right\}$, takes values in $C([0 ; 1])$ and satisfies the condition $f(r, 0)=r, r \in \mathbb{Q}_{2}$. Such functions are in one to one correspondence with the sequences $\left\{f_{n} ; n \geq 1\right\}$ from (6.1). If $I(f)<+\infty$, then for arbitrary $r_{1} \leq r_{2}$ from $\mathbb{Q}_{2}$ and $t \in[0 ; 1]$

$$
f\left(r_{1}, t\right) \leq f\left(r_{2}, t\right) .
$$

For $f$ satisfing (6.2) and $r_{1}<r_{2}$ from $\mathbb{Q}_{2}$ define

$$
\tau\left(r_{1}, r_{2}\right)=\inf \left\{t: f\left(r_{1}, t\right)=f\left(r_{2}, t\right)\right\} \wedge 1 .
$$

Then for every $n \geq 1$

$$
I_{n}(f)=\frac{1}{2} \sum_{k=0}^{2^{n}} \int_{0}^{\tau\left(\frac{k-1}{2^{n}}, \frac{k}{2^{n}}\right)} \dot{f}\left(\frac{k}{2^{n}}, s\right)^{2} d s,
$$

where

$$
\tau\left(\frac{-1}{2^{n}}, 0\right)=1
$$


Note that $\left\{I_{n}(f) ; n \geq 1\right\}$ is nondecreasing and

$$
I(f)=\lim _{n \rightarrow \infty} I_{n}(f) .
$$

Define the space of trajectories for Arratia's flow. Denote by $\mathcal{M}$ the set of real-valued functions defined on $[0 ; 1]^{2}$, which have the following properties:

1) for every $u \in[0 ; 1] y(u, \cdot) \in C([0 ; 1])$,

2) for all $u_{1} \leq u_{2}, t \in[0 ; 1]$

$$
y\left(u_{1}, t\right) \leq y\left(u_{2}, t\right),
$$

3) for every $t \in[0 ; 1] y(\cdot, t)$ is a càdlàg function,

4) for all $u_{1}, u_{2} \in[0 ; 1]$

$$
\begin{gathered}
y\left(u_{1}, t\right)=y\left(u_{2}, t\right), t \geq \tau_{u_{1} u_{2}}, \\
\tau_{u_{1} u_{2}}=\inf \left\{s: y\left(u_{1}, s\right)=y\left(u_{2}, s\right)\right\},
\end{gathered}
$$

5) for every $u \in[0 ; 1]$

$$
y(u, 0)=u .
$$

An arbitrary element of $\mathcal{M}$ can be treated as a continuum forest. We will endow $\mathcal{M}$ with the distance

$$
\rho\left(y_{1}, y_{2}\right)=\max _{t \in[0 ; 1]} \sigma\left(y_{1}(\cdot, t), y_{2}(\cdot, t)\right),
$$

where $\sigma$ is the Lévy-Prokhorov distance between càdlàg functions. Note that the convergence of $y$ in the distance $\sigma$ for a fixed $t$ is equivalent to the weak convergence of $\mu_{t}$. Now define the subset $\mathcal{R}$ of $C([0 ; 1])^{\infty}$ as follows. $\mathcal{R}$ consists of real-valued functions defined on $\mathbb{Q}_{2} \times[0 ; 1]$, which have the properties:

1) for every $r \in \mathbb{Q}_{2}$

$$
y(r, \cdot) \in C([0 ; 1]),
$$

2) for all $r_{1} \leq r_{2}, t \in[0 ; 1]$

$$
y\left(r_{1}, t\right) \leq y\left(r_{2}, t\right)
$$

3) for every $r \in \mathbb{Q}_{2}$

$$
y(r, 0)=r .
$$

Note that $\mathcal{R}$ as a subset of $C([0 ; 1])^{\infty}$ is closed in the distance of pointwise convergence on $\mathbb{Q}_{2}$ and uniform convergence on $[0 ; 1]$. 
Consider a map $i: \mathcal{R} \rightarrow \mathcal{M}$ which is defined as follows $\forall u, t \in[0 ; 1], y \in \mathcal{R}:$

$$
i(y)=\widetilde{y}, \widetilde{y}(u, t)=\inf _{\substack{r \in \mathbb{Q}_{2} \\ r>u}} y(r, t) .
$$

Lemma 6.1. $i$ is continuous mapping.

Proof. Suppose, that $y_{n} \rightarrow y, n \rightarrow \infty$ in $\mathcal{R}$. For a given positive $\varepsilon$ consider a partition $0=r_{0}<\ldots<r_{m}=1$ consisting of the points from $\mathbb{Q}_{2}$ and satisfying the property

$$
\max _{k=0, \ldots, m-1}\left(r_{k+1}-r_{k}\right)<\frac{\varepsilon}{2} .
$$

Take $n$ such, that

$\forall k=0, \ldots, m$ :

$$
\max _{[0 ; 1]}\left|y_{n}\left(r_{k}, t\right)-y\left(r_{k}, t\right)\right|<\frac{\varepsilon}{2} .
$$

Consider an arbitrary $u \in[0 ; 1)$. Then there exists $r_{k} \in(u, u+\varepsilon)$. For such $r_{k}$ and arbitrary $t \in[0 ; 1]$

$$
\begin{aligned}
\widetilde{y}_{n}(u, t) & \leq y_{n}\left(r_{k}, t\right) \leq y\left(r_{k}, t\right)+\frac{\varepsilon}{2} \leq \\
& \leq \widetilde{y}(u+\varepsilon, t)+\frac{\varepsilon}{2},
\end{aligned}
$$

and

$$
\widetilde{y}(u, t) \leq y\left(r_{k}, t\right) \leq y_{n}\left(r_{k}, t\right)+\frac{\varepsilon}{2} \leq \widetilde{y}_{n}(u+\varepsilon, t)+\frac{\varepsilon}{2} .
$$

Now take $u \in[\varepsilon ; 1]$. There exists $r_{k} \in(u-\varepsilon, u)$. For such $r_{k}$ and arbitrary $t \in[0 ; 1]$

$$
\widetilde{y}_{n}(u, t) \geq y_{n}\left(r_{k}, t\right) \geq y\left(r_{k}, t\right)-\frac{\varepsilon}{2} \geq \widetilde{y}(u-\varepsilon, t)-\frac{\varepsilon}{2},
$$

and

$$
\widetilde{y}(u, t) \geq y\left(r_{k}, t\right) \geq y_{n}\left(r_{k}, t\right)-\frac{\varepsilon}{2} \geq \widetilde{y}_{n}(u-\varepsilon, t)-\frac{\varepsilon}{2} .
$$

The lemma is proved.

As a consequence of the previous lemma and the contraction principle we obtain the LDP for Arratia's flow. 
Theorem 6.1. Let $\left\{x^{\varepsilon}(u, t)=x(u, \varepsilon t), u \in(0 ; 1), t \in[0 ; 1]\right\}, \varepsilon \in(0 ; 1)$ are the random fields obtained from Arratia's flow by the time changing. Then the family $\left\{x^{\varepsilon}\right\}_{\varepsilon>0}$ satisfies the LDP in $\mathcal{M}$ with the rate function

$$
I(x)=\inf _{i(h)=x} I_{0}(h),
$$

where $I_{0}(h)$ is given by (6.1).

\section{References}

[1] Darling R.W.R. Constructing of nongomeomorphic stochastic flows. Mem. Amer. Math. Soc., 70, No. 376, 1987. - 97 p.

[2] Le Jan Y., Raimond O. Flows, coalescence and noise. - Ann. Probab. 32, No. 2, 2004. - 1247-1315 p.

[3] Kunita H. Stochastic flows and stochastic differential equations. - Cambridge Studies in Advanced Mathematics, 24, Cambridge University Press, Cambridge, 1997. - 346 p.

[4] Liptser R.Sh., Shyriaev A.N. Theory of Martingales. - Math. Appl., Kluwer, Dordrecht, 1989. - 808 p.

[5] Dorogovtsev A.A. One Brownian stochastic flows. - Theory of Stochastic Processes. - 10(26), No. 3-4, 2004. - 21-25 p.

[6] Budhiraja A., Dupuis P., Maroulas V. Large Deviations for Stochastic Flows of Diffeomorphisms. Submitted for Publication.

[7] Budhiraja A., Dupuis P., Maroulas V. Large Deviations for infinite dimensional dynamical systems. - Ann. Prob., 36, no.4, 2008. - 1390$1420 \mathrm{p}$.

[8] Budhiraja A., Dupuis P. A variational representation for positive functional of infinite dimensional Brownian motions. - Probability and Mathematical Statistics, 20, 2000. - 39-61 p.

[9] Dembo A., Zeitouni O. Large deviations techniques and applications. N.Y.: Springer Verlag, 1998. - 408 p. 
[10] Bogachev V.I. Gaussian measures. - AMS, 1998. - 433 p.

[11] Lifshits M.A. Gaussian random function. - Math. Appl., Kluwer, Dordrecht, 1995. - 333 p.

[12] Ikeda N., Watanabe S. Stochastic differential equations and diffusion processes. - North-Holland Publishing Co., Amsterdam; Koconsha, Ltd., Tokyo, 1989. - 555 p.

[13] Dorogovtsev A. A. Some remarks on a Wiener flow with coalescence. (Russian) translation in Ukrainian Math. J. 57, no. 10, 2005. - 1550-1558 p.

[14] Dorogovtsev A. A. A stochastic integral with respect to Arratia's flow.(Russian) Dokl. Akad. Nauk, 410, no. 2, 2006. - 156-157 p.

[15] Dorogovtsev A. A. Meroznachnye protsessy i stokhasticheskie potoki. (Russian) [Measure-valued processes and stochastic flows], Kiev: Institute of Math. NANU, 2007. - 290 p.

[16] Peitgen H.O., Saupe D. The science of Fractal Image. - N.Y.: Springer Verlag, 1988. - $312 \mathrm{p}$.

[17] Chen, Mu Fa, From Markov chains to nonequilibrium particle systems. - World Scientific Publishing Co., Inc., River Edge, NJ, 1992. - 550 p.

[18] Kallenberg O. Foundations of modern probability. - Second edition. Probability and its Applications. N.Y.: Springer Verlag, 2002. - 638 p. 